HIP-2009-32/TH

\title{
Inhomogeneous Structures in Holographic Superfluids: II. Vortices
}

\author{
Ville Keränen, ${ }^{1,2} *$ Esko Keski-Vakkuri, 讯 Sean Nowling, ${ }^{1,3}$ K K. P. Yogendran ${ }^{1,4} \S$ \\ ${ }^{1}$ Helsinki Institute of Physics \\ P.O.Box 64, FIN-00014 University of Helsinki, Finland \\ ${ }^{2}$ Department of Physics \\ P.O.Box 64, FIN-00014 University of Helsinki, Finland \\ ${ }^{3}$ Department of Mathematics and Statistics \\ P.O.Box 68, FIN-00014 University of Helsinki, Finland \\ ${ }^{4}$ IISER Mohali \\ MGSIPAP Complex, Sector 26 Chandigarh 160019 , India
}

\begin{abstract}
We study vortex solutions in a holographic model of Herzog, Hartnoll, and Horowitz, with a vanishing external magnetic field on the boundary, as is appropriate for vortices in a superfluid. We study the relevant length scales related to the vortices and how the charge density inside the vortex core behaves as a function of temperature or chemical potential. We extract a critical superfluid velocity from the vortex solutions, study how it behaves as a function of the temperature, and compare it to earlier studies and to the Landau criterion. We also comment on the possibility of a Berezinskii-Kosterlitz-Thouless vortex confinement-deconfinement transition.
\end{abstract}

*ville.keranen@helsinki.fi

†esko.keski-vakkuri@helsinki.fi

${ }^{\ddagger}$ sean.nowling@helsinki.fi

§yogendran.kalpat@helsinki.fi 


\section{Introduction}

One of the focal points of activity in applying holographic methods to condensed matter systems has been studies of holographic superconductors. An initial spark was the observation in [1, where it was noted that an Abelian Higgs model coupled to gravity in AdS space exhibits spontaneous symmetry breaking of the local $U(1)$ symmetry (in the bulk) as the charge of the black hole was increased. In [2], this phase transition was interpreted in the dual field theory as a superconducting phase transition, since in the model a charged operator gets a vacuum expectation value $(\mathrm{VEV})$.

In this paper we take a more conservative stance and use the superfluid interpretation [3, 4]. We are continuing our investigation of inhomogeneous extended configurations in the model [2], which we begun in [5, 6] reporting on holographical dark solitons. In this paper we show that the model [2] allows vortex solutions even in the absence of a magnetic field on the boundary. In this case, the superfluid interpretation is most natural: in a superconductor a vortex would act as a source for a dynamical magnetic field and require turning on a flux line, whereas we are explicitly setting the boundary $\vec{B}=0$ and yet find a consistent solution. Droplet and vortex solutions in the presence of boundary magnetic fields have previously been discussed in [7-9].1] An early work on holographic vortices is a construction of a vortex line in pure AdS space [14] and in the AdS-Schwarzschild background [15].

We construct vortex solutions in [2], by solving the equations of motion of the Abelian Higgs model in the probe approximation, i.e. neglecting the backreaction of the scalar field and the gauge fields on the black hole geometry. We study basic properties of the vortex solutions, such as the free energy and associated length scales. We also study the amount of density depletion in the core of the vortex, and find agreement with earlier studies [5, 6]. In [6] we noted that the density depletion in the core of a dark soliton depends on the dimension of the condensing operator in a way reminiscent to a loosely bound fermion pair for dimension $\Delta=2$ and a more tightly bound fermion pair for $\Delta=1$, by comparing to an earlier study in nonrelativistic superfluids [16]. For vortices a similar study of the depletion fraction in a non-relativistic setting is in [17].

In this work we extend the analogy between the type of the superfluid and the dimension of the condensing operator by studying critical superfluid velocities above which the superfluid flow starts to dissipate and superfluidity is ruined. The critical velocity is defined from the core size of the vortices. Our results for critical flows partially overlap with others obtained from constant superfluid flow in [3, 4].

We also compare the critical velocity of superfluid flow to velocity of second sound (the second sound velocity was calculated in [3,18] using different techniques), in the

\footnotetext{
${ }^{1}$ For other studies of external magnetic fields on holographic superconductors see for example [10 13 .
} 
spirit of Landau criterion, and find that for both scaling dimensions the superfluid velocity is below the sound velocity. The proposal that superfluidity in the $\Delta=2$ case might be due to loosely bound fermions is partly supported by the fact that the critical velocity is not set by the superfluid's second sound.

The outline of the paper goes as follows. In section 2 we introduce the model and use cylindrical symmetry to derive the form of the vortex solution, and the corresponding equations of motion. In sections 3 and 4 we describe our numerical methods of solving the partial differential equations. In sections 5 and 6 we study the characteristic length scales of the vortex and the density depletion. Section 7 discusses the critical velocity as determined from the vortices. In section 8 we show that the free energy of one vortex is log divergent as is the case in superfluids (a related calculation has been done in [8]), and we study how the coefficient of the $\log$ term behaves as a function of temperature and comment on the possibility of a Berezinskii-Kosterlitz-Thouless (BKT) phase transition [19,20] in the system. Finally we end with a discussion and outline future directions.

\section{Review of superfluids and vortices}

We will begin by reviewing some basic properties of superfluids in $2+1$ dimensions and how vortices arise in them and what role they play. A superfluid is characterized by spontaneous breaking of a global $U(1)$ symmetry. This already poses a theoretical puzzle in $2+1$ dimensions since such spontaneous symmetry breaking at finite temperature should not be possible due to the Mermin-Wagner theorem. In the holographic model, this problem is perhaps being circumvented by having a large number $N$ of degrees of freedom in the field theory 2 . In this section, we will assume that spontaneous symmetry breaking is stabilized in the large- $N$ limit and we will look at the consequences of that for the low energy physics of the superfluid.

The low lying excitations in a superfluid are the Goldstone bosons from spontaneous breaking of the $U(1)$ symmetry. At large length scales, as compared to the inverse mass of the lightest massive quasiparticle, the system is well described by an effective action for the Goldstone field $\phi(x)$. At zero chemical potential where the effective action is manifestly Lorentz invariant, the only non-irrelevant operator one can write down is $\left(\partial_{\mu} \phi\right)^{2}$. Generalizing this to a finite chemical potential, $\mu$, can be done by introducing a formal gauge field $A_{\mu}$ with one non-zero component, $A_{0}=\mu\left[29\right.$. Since the effective action respects the formal gauge invariance $\phi \rightarrow e^{i \Lambda} \phi$,

\footnotetext{
${ }^{2}$ See additional discussion in $[21+23]$. The question of identifying the $N$ degrees of freedom can be addressed after embedding holographic model into string/M theory see 24 28 , for work in this direction.
} 
$A_{\mu} \rightarrow A_{\mu}+\partial_{\mu} \Lambda$ it has the form

$$
S_{e f f}=-\int d^{3} x \frac{1}{2} \kappa\left(D_{\mu} \phi\right)^{2}=\int d^{3} x \frac{1}{2} \kappa\left(\left(\partial_{0} \phi-\mu\right)^{2}-\left(\partial_{i} \phi\right)^{2}\right)
$$

The action (11) has a conserved momentum density $T_{0 \mu}=\kappa \partial_{0} \phi \partial_{\mu} \phi$ and a current density $j_{i}=\kappa \partial_{i} \phi$. Both of the four vectors are parallel to $\partial_{\mu} \phi$, which motivates one to define the superfluid velocity vector $v_{s}^{\mu}=\gamma \partial^{\mu} \phi$. The coefficients $\kappa$ and $\gamma$ seem at the moment to be arbitrary, but for a relativistic superfluid they can be connected to thermodynamic quantities as [30]

$$
\kappa=\frac{\rho_{s}}{\mu}, \quad \gamma=\frac{1}{\mu},
$$

where $\rho_{s}$ is the superfluid density. These relations will be important in later sections, so that we will derive them in a simple example in the next section.

We can define a vortex as a state for which the expectation value of the superfluid velocity $\left\langle v_{s}^{i}\right\rangle=\left\langle\partial^{i} \phi\right\rangle / \mu$ is cylindrically symmetric and has a non-zero circulation

$$
c=\mu \oint d x_{i}\left\langle v_{s}^{i}\right\rangle
$$

where the integral is taken over circle of constant radius. Using the averaged (time independent) Heisenberg equation

$$
0=\left\langle\partial_{i} \partial^{i} \phi\right\rangle=\mu \partial_{i}\left\langle v_{s}^{i}\right\rangle
$$

we see that the radial part of the superfluid velocity is a constant. We will focus on the case where this constant vanishes. Using (3) we get the superfluid velocity of the vortex as

$$
\left\langle v_{s}^{i}\right\rangle=c \epsilon^{i j} \frac{x^{j}}{\rho^{2}}
$$

where $\rho$ is the radial coordinate on two dimensions. For a normal fluid the circulation $c$ can take any values, but in a superfluid $\phi$ is the phase of a complex field and thus, the circulation is quantized in integer multiplets of $2 \pi$. Quantized circulation is one of the hallmarks of superfluid vortices.

Even though it is easy to see the existence of vortices simply by topological arguments and/or by use of the simple Goldstone effective action, the interior of the vortex is model dependent and carries interesting information about the microscopic structure of the superfluid [16]. Since the superfluid velocity diverges inside the vortex core, the vortex solution must interpolate all the way from the superfluid to the normal phase, with $\rho_{s}=0$, as a function of $\rho$. Thus, in order to describe a vortex, one is forced to appeal to a microscopic description that is able to describe the normal phase. 
The energy of a single vortex can be estimated from the effective action (11) as

$$
E_{\text {vor }} \approx \int d^{2} x \frac{1}{2} \mu \rho_{s}\left(\left\langle v_{s}\right\rangle\right)^{2} \approx \frac{\pi \rho_{s} n^{2}}{\mu} \log \left(\frac{R_{c}}{\xi}\right),
$$

where $R_{c}$ is an IR cutoff and $\xi$ a UV cutoff. In a microscopic description the UV divergence is absent since $\rho_{s}$ vanishes at the vortex core, so we are left with an IR divergence in the vortex energy. This does not still mean that vortices would not exist in superfluids. Vortices will be formed in a superfluid when the system is put under rotation with angular frequency $\omega$, which is larger than a critical frequency $\omega_{c}$ (see eg. [31]). If the size of the system is taken to infinity, the critical angular velocity tends to zero and vortices will immediately form when the system is rotating. Another scenario where vortices are important is during the BKT transition. In that situation, the entropy of a vortex becomes comparable to $E_{v o r} / T$ and it becomes thermodynamically preferred to nucleate vortices in the system.

The divergence of the vortex energy is in accordance with Derrick's theorem [32, which states that the energy of a soliton solution in a theory with only scalar fields (with canonical kinetic terms) is divergent if the space dimension of the system $D$ is larger than 1. In the holographic setting, there are also gauge fields involved, so that Derrick's theorem as such does not apply, and thus it is not a priori clear, whether the vortex energy is finite or not, in the holographic model. But as we will see, the vortex energy is indeed logarithmically divergent (for a related calculation see [8]). This is necessary for the superfluid phase to exist in non-zero temperature, since if it would take finite energy to create a vortex, it would be entropically favorable at any temperature to nucleate a soup of vortices, which would drive the order parameter to zero.

\subsection{An example model with vortices: The Gross-Pitaevskii equation}

To get some insight into the microscopic structure of superfluid vortices, before attacking the holographic problem, we study vortices in the relativistic Gross-Pitaevskii (GP) equation (we will drop the label "relativistic" in what follows). The GP equation can be thought of as arising from a saddle point evaluation of a path integral for the bosonic field $\Psi$, with the grand canonical action

$$
S_{G P}=\int d^{3} x\left(\left|\partial_{0} \Psi-i \mu \Psi\right|^{2}-\left|\partial_{i} \Psi\right|^{2}-V|\Psi|^{2}-\frac{1}{2} g|\Psi|^{4}\right) .
$$

The GP equation is relevant for weakly interacting, dilute Bose-Einstein (BEC) condensates and tightly bound fermionic superfluids at low temperatures.

It is interesting to see how one can end up on an effective action of the form (11) from the GP theory. This gives a simple example where one can derive the 
identifications (2) easily. By integrating out the fluctuations of the modulus in (7) we end up with an effective action for the Goldstone field, of the form

$$
S_{e f f}=\int d^{3} x\left|\Psi_{0}\right|^{2}\left(\left|\partial_{0} \phi-\mu\right|^{2}-\left|\partial_{i} \phi\right|^{2}\right)
$$

where $\Psi_{0}$ is the VEV of $\Psi$. This action is clearly of the form (11). In the GP equation the superfluid density is simply the particle number density derived from the action (17), $\rho_{s}=\frac{-i}{2}\left(\Psi^{*} \partial_{t} \Psi-\Psi \partial_{t} \Psi^{*}+2 i \mu|\Psi|^{2}\right) \approx \mu\left|\Psi_{0}\right|^{2}$, where in the second equality we have expanded around the ground state and ignored fluctuations. Thus the effective action becomes

$$
S_{\text {eff }}=\int d^{3} x \frac{\rho_{s}}{\mu}\left(\left|\partial_{0} \phi-\mu\right|^{2}-\left|\partial_{i} \phi\right|^{2}\right),
$$

which is the same thing as (1) with $\kappa=\rho_{s} / \mu$. The particle number current is now $j_{i} \approx\left|\Psi_{0}\right|^{2} \partial_{i} \chi=\rho_{s} \partial_{i} \chi / \mu \equiv \rho_{s} v_{s}$, which tells us that $v_{s}=\partial_{i} \chi / \mu$. Thus we see how the identification (2) arises from relativistic GP theory.

Let us proceed to discuss vortices. For a time independent field configuration the GP equation is

$$
-\nabla^{2} \Psi+\left(V-\mu^{2}\right) \Psi+g|\Psi|^{2} \Psi=0 .
$$

Note that the non-relativistic version of the time independent GP equation can be obtained from (10) simply by replacing $\mu^{2}$ by $\mu$. An ansatz for a vortex solution can be taken in the form $\Psi=e^{i n \theta} R(\rho)$, where $n$ is the winding number (or circulation) of the vortex. Plugging the ansatz into the GP equation gives

$$
-R^{\prime \prime}-\frac{1}{\rho} R^{\prime}+\frac{n^{2}}{\rho^{2}} R+\left(V-\mu^{2}\right) R+g R^{3}=0 .
$$

Again the superfluid velocity has the same form as in (5). Since (11) does not have known analytic solutions for non-zero winding $n$, we will solve it numerically. The numerical solution is displayed in Fig 1. From the numerics it seems that the field $R(r)$ behaves in a power law fashion at large values of $\rho$. This suggests that we can try to get an analytic solution for asymptotically in $\rho$. Plugging a power series ansatz to (11) gives the leading behavior

$$
R(\rho)=\sqrt{\frac{\mu^{2}-V}{g}}\left(1-\frac{n^{2} \xi_{2}^{2}}{2 \rho^{2}}+\ldots\right),
$$

and the particle number density (which is equivalent to the superfluid density) is given by

$$
\rho_{s}(\rho)=\mu R^{2}(\rho)=\mu \frac{\mu^{2}-V}{g}\left(1-\frac{n^{2} \xi_{2}^{2}}{\rho^{2}}+\ldots\right),
$$

where $\xi_{2}=1 / \sqrt{\mu^{2}-V}$. For the unit winding vortex we can also define a length scale from the vortex's core, $R \approx R(\infty)\left(\rho / \xi_{1}+\ldots\right)$. We can numerically calculate $\xi_{1}$, which 


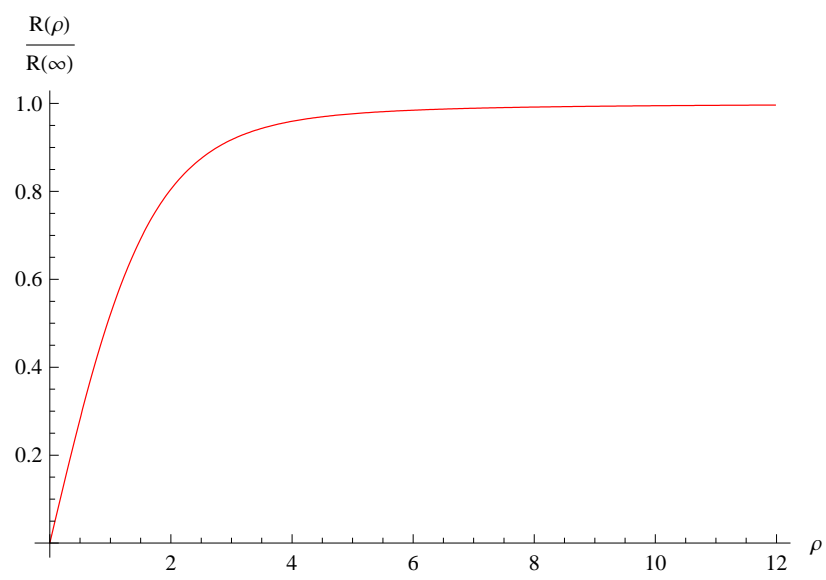

Figure 1: Spatial profile of a vortex solution to the GP equation.

gives the ratio

$$
\frac{\xi_{1}}{\xi_{2}} \approx 1.75
$$

Note that there is really only one length scale in the problem. This means that at sufficiently low temperatures (that is, when the GP equation is valid) the ratio (14) is a constant as the chemical potential is varied. We will revisit this in the context of holographic superfluids in section 5 .

\section{The model}

As argued in [2], a holographic dual of a superfluid is provided by a scalar field coupled to an Einstein-Maxwell system in asymptotically $A d S$ space.

$$
S_{A d S}=\int d^{4} x \sqrt{-g}\left[\frac{1}{\kappa_{4}^{2}}\left(\mathcal{R}-\frac{6}{L^{2}}\right)-\frac{1}{q^{2}}\left(\left|D_{\mu} \Psi\right|^{2}+m^{2}|\Psi|^{2}+\frac{1}{4} F_{\mu \nu}^{2}\right)\right],
$$

As in [2], will choose the mass $m^{2}=-2 / L^{2}$. Throughout this work we will work in the probe limit, $\left(\frac{\kappa_{4}^{2}}{q^{2}}\right.$ is small $)$, so that the backreaction to gravity can be ignored. Thus, we are working with the fixed bulk metric

$$
d s^{2}=\frac{L^{2}}{z^{2}}\left(-f(z) d t^{2}+f(z)^{-1} d z^{2}+d \rho^{2}+\rho^{2} d \theta^{2}\right)
$$

where $f(z)=1-\frac{z^{3}}{z_{T}^{3}}$. The AdS-CFT dictionary tells us that

$$
e^{-S_{\text {AdS }}(\text { onshell })}=\left\langle e^{-\int d^{3} x\left(\rho(x) \mu(x)+\mathcal{O}_{1}(x) \mathcal{O}_{2}(x)\right)}\right\rangle_{Q F T},
$$

where the boundary quantum field theory operators are related to the boundary values of the bulk AdS fields by the relations

$$
\Psi(x, z)=z \mathcal{O}_{1}(x)+z^{2} \mathcal{O}_{2}(x)+\ldots \quad A_{t}(x, z)=\mu(x)+z \rho(x)+\ldots
$$


Thus, in order to find the QFT operator expectation values we need to solve the classical equations of motion in AdS space to obtain the on shell fields. Ignoring gravitational backreaction, the equations of motion become

$$
\begin{aligned}
& 0=\frac{1}{\sqrt{-g}} \partial_{\mu}\left(\sqrt{-g} g^{\mu \nu} \partial_{\nu} R\right)+m^{2} R-R\left(\partial_{\mu} \chi-A_{\mu}\right)^{2} \\
& 0=\frac{1}{\sqrt{-g}} \partial_{\mu}\left(\sqrt{-g} F^{\mu \nu}\right)-R^{2}\left(A^{\nu}-\partial^{\nu} \chi\right) \\
& 0=\partial_{\mu}\left(\sqrt{-g} R^{2} g^{\mu \nu}\left(\partial_{\nu} \chi-A_{\nu}\right)\right)
\end{aligned}
$$

where we have defined the real valued fields $R$ and $\chi$ according to the relation $\Psi=$ $\frac{1}{\sqrt{2}} R e^{i \chi}$.

The equations of motion may be greatly simplified using the observation that cylindrical symmetry implies that gauge invariant quantities are independent of $\theta$. A detailed discussion of gauge fixing and the use of symmetry is presented in Appendix A. An important point discussed in Appendix $B$ is that the field $\chi$ is a normalizable mode. If $\chi$ was non-normalizable, its boundary value would be set by hand, which in turn would imply that features such as the superfluid velocity profile are not determined by the system dynamically.

Working in the $A_{z}=0$ gauge and defining the field $\tilde{R}=R / z$, the equations which describe a vortex profile are

$$
\begin{aligned}
0 & =f \partial_{z}^{2} \tilde{R}+\partial_{z} f \partial_{z} \tilde{R}-z \tilde{R}+\frac{1}{\rho} \partial_{\rho}\left(\rho \partial_{\rho} \tilde{R}\right) \\
& -\tilde{R}\left(-\frac{1}{f} A_{t}^{2}+\frac{\left(A_{\theta}-n\right)^{2}}{\rho^{2}}\right) \\
0 & =f \partial_{z}^{2} A_{t}+\frac{1}{\rho} \partial_{\rho}\left(\rho \partial_{\rho} A_{t}\right)-\tilde{R}^{2} A_{t} \\
0 & =\partial_{z}\left(f \partial_{z} A_{\theta}\right)+\rho \partial_{\rho}\left(\frac{1}{\rho} \partial_{\rho} A_{\theta}\right)-\tilde{R}^{2}\left(A_{\theta}-n\right),
\end{aligned}
$$

where all the fields are functions of only $z$ and $\rho$. As discussed in appendix $\AA$ the only non-zero gauge field components are $A_{t}$ and $A_{\theta}$. 3

\footnotetext{
${ }^{3}$ Since there is a magnetic flux $\int_{z=z_{0}} F \neq 0$ going through the vortex core in the bulk, one can ask what happens to it as one approaches the boundary. Since the magnetic flux is conserved $\int_{\Sigma} F=0$ for any closed surface $\Sigma$, the magnetic flux has to go somewhere as one approaches the boundary. Since we have a vanishing boundary magnetic field the magnetic flux does not reach the boundary, but rather it turns to the $\rho$ direction in the form of the magnetic field $B_{\rho} \sim 1 / \rho$. In the holographic dictionary $B_{\rho}=\partial_{z} A_{\theta} / \rho$ is not identified as a magnetic field, but rather with the superfluid current $j_{\theta}$. So, in short, the magnetic flux turns into superfluid flow on the boundary.
} 


\section{Finding the vortex solutions}

\subsection{The method}

The equations of motion are a set of coupled nonlinear partial differential equations, which do not seem to be solvable analytically. In order to proceed, we therefore resort to numerical methods. Because the vortex solutions are inhomogeneous, it is difficult to use standard differential equation solvers, such as Mathematica's NDSolve. Instead we will use a Gauss-Seidel relaxation scheme. 4 As described in [5, 6], we first place the system on a finite box of radius $L,(z, \rho) \in[0,1] \times[0, L]$ and discretize along both axes. After the usual procedure of replacing derivatives with finite difference derivatives, the differential equations are turned into finite difference equations.

The resulting finite difference equations are solved by choosing an initial seed configuration and iterating with the Gauss-Seidel method. The error in solving the equations of motion falls off with a power law as the lattice size is increased. With a sufficient number of iterations and a fine enough lattice, the seed configuration relaxes to a solution to the equations of motion. For further details on this numerical method see [6].

\subsection{Boundary conditions}

To find a unique solution to the finite difference equations, we specify boundary conditions at each edge of the region being simulated. The boundary conditions at the AdS boundary determine the external sources turned on in the dual field theory. The gauge field, $A_{t}$, takes a constant value (independent of $\rho$ and $\theta$ ) on the boundary corresponding to a constant chemical potential, $A_{t}(z=0)=\mu$. The scalar field, $\tilde{R}=|\Psi| /(\sqrt{2} z)$, can satisfy either Dirichlet $\tilde{R}(z=0)=0$ or Neumann $\partial_{z} \tilde{R}(z=0)=0$ boundary conditions, corresponding to two different boundary theories with different scaling dimensions of the condensing scalar operator, either $\mathcal{O}_{2}$ or $\mathcal{O}_{1}$. Finally the angular gauge field, $A_{\theta}$, satisfies Dirichlet boundary conditions, $A_{\theta}(z=0)=0$. This is because we want the boundary magnetic field to vanish, implying $\partial_{\rho} A_{\theta}(z=0)=0$. Because the boundary value of $A_{\theta}$ is simply a constant, we can choose such a gauge that $A_{\theta}(z=0)=0$.

On the $\rho=L$ boundary of the region being simulated we impose Neumann boundary conditions for all the fields, since we want the fields to asymptote to the translationally invariant symmetry breaking solution. We have verified that the solutions and results in this paper are unchanged when one increases $L$, hence we can safely conclude that we have used a large enough box.

\footnotetext{
${ }^{4}$ In Appendix C we discuss a complementary approach valid away from the vortex core. In that regime, the differential equations reduce to ordinary differential equations and one may use "off the shelf" tools.
} 
Since the core of the vortex should be in the normal phase we impose Dirichlet boundary conditions on the scalar field $\tilde{R}(\rho=0)=0$ in the core. Because the scalar field vanishes in the origin, there is no bulk electric charge at the vortex core. Therefore, along the $\rho=0$ boundary we impose Neumann boundary conditions for the gauge field $A_{t}$ such that center of the vortex is not a source for the radial electric field. This is basically a regularity condition. Finally we impose the Dirichlet boundary condition $A_{\theta}(\rho=0)=0$ on the angular gauge field. This may also be regarded as a regularity conditions along the vortex core. We want the bulk magnetic field $B_{z}=\partial_{\rho} A_{\theta} / \rho$ to be finite at the origin. This means that $A_{\theta}$ has the behavior $A_{\theta}=$ $A_{\theta}^{(0)}(z)+\rho^{2} A_{\theta}^{(1)}(z)$ near the vortex core. To have finite superfluid current in the core we require the $\partial_{z} A_{\theta} / \rho$ to be finite in the limit $\rho \rightarrow 0$. This means the $\partial_{z} A_{\theta}^{(0)}=0$. Furthermore, since we required $A_{\theta}(z=0)=0$ fixes the constant part $A_{\theta}^{(0)}=0$ and, thus $A_{\theta}(\rho=0)=0$.
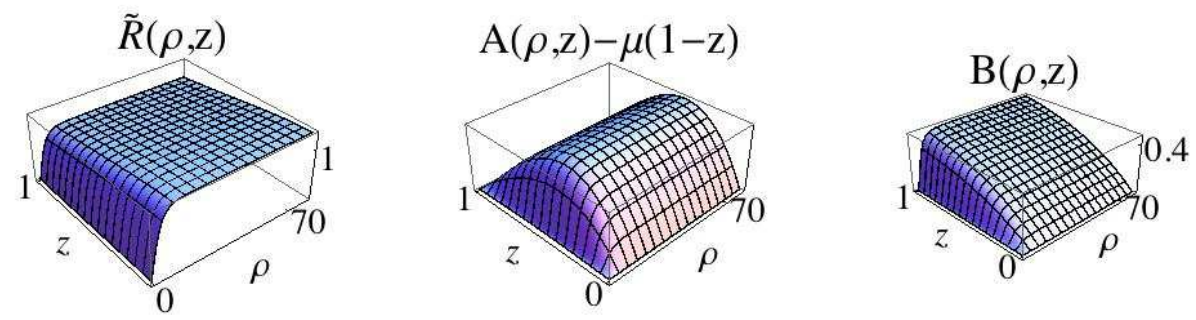

Figure 2: Typical field profiles for the $\left\langle\mathcal{O}_{1}>\right.$ condensate. (For visualization purposes we have subtracted a linear background from $A$.)
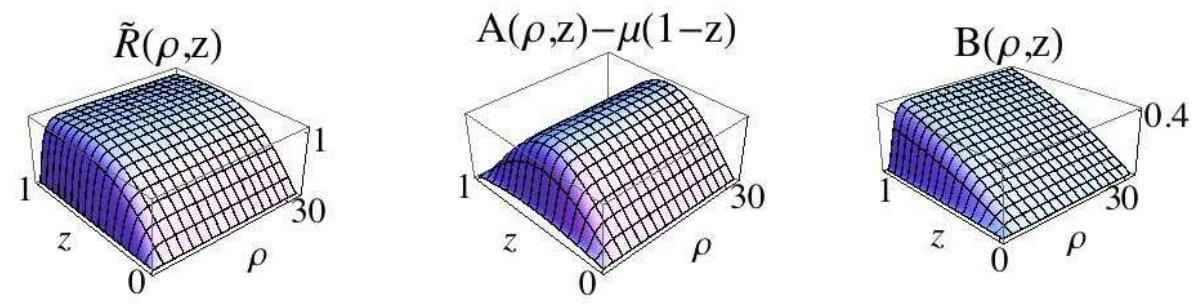

Figure 3: Typical field profiles for the $<\mathcal{O}_{2}>$ condensate. (For visualization purposes we have subtracted a linear background from $A$.)

The final boundary conditions to discuss are regularity conditions to be imposed along the black hole horizon. The equations of motion are elliptic outside the horizon, 
but become parabolic along the horizon. Also, we require that $A_{t}(z=1)=0$ so that the connection one form be normalizable at the horizon. This leads to the following regularity conditions at the horizon.

$$
\begin{aligned}
& 0=\left(\partial_{z} f\right) \partial_{z} \tilde{R}-\tilde{R}+\frac{1}{\rho} \partial_{\rho}\left(\rho \partial_{\rho} \tilde{R}\right)-\tilde{R} \frac{\left(A_{\theta}-n\right)^{2}}{\rho^{2}} \\
& 0=A_{t} \\
& 0=\left(\partial_{z} f\right) \partial_{z} A_{\theta}+\rho \partial_{\rho}\left(\frac{1}{\rho} \partial_{\rho} A_{\theta}\right)-\tilde{R}^{2}\left(A_{\theta}-n\right) .
\end{aligned}
$$

\subsection{Existence and stability}

Vortex solutions of (22)-(24) are topologically stable, with a conserved topological charge

$$
n=\int_{0}^{2 \pi} d \theta \partial_{\theta} \chi .
$$

For all regular solutions $n$ is an integer, and thus continuous time evolution cannot change its value.

If one prepares the system in a configuration of non-zero winding, the time evolution will keep the field in the same winding sector for all times. Eventually the system

will stabilize to the minimum energy field configuration which solves the equations of motion with vanishing time derivatives. They are simply the static equations $(22),(23)$, and (24). The minimum energy field configuration is approximated by the solutions we find numerically.

We are solving the equations using a relaxation method, which means that the solution evolves in the iteration time $\tau$ by a (generalized) diffusion equation. In order to establish that our numerical solutions approach the minimum energy solution in the desired winding sector, we need to show that the field $\tilde{R}$ does not generate a discontinuity to "unwind" the solution. The fact that this does not happen follows from the diffusive nature of the iteration algorithm. The diffusive nature guarantees that the energy of a configuration is non-increasing. Thus, if we start from a seed configuration with finite energy density above the ground state, we will not generate discontinuities to the fields since they would require too much energy.

\section{Characteristic scales}

Typical condensate, charge density, and superfluid density radial profiles as extracted from the bulk solutions, are shown in Fig.(4).

We begin our analysis of the vortices described in section (4.1) by focusing on unit winding vortices. The first quantities we would like to identify in these vortices are the relevant scales of variation. It can be seen from our numerical solutions that the 
fields have a power-law fall off as $\rho \rightarrow \infty$. This fact is confirmed in the tail regime as is discussed in appendix C.

Using the standard AdS/CFT dictionary, the behavior of the condensate may be read off from the boundary behavior of the scalar field. In this way we can define a coherence length by either fitting a power-law function of the form

$$
\left\langle\mathcal{O}_{i}(\rho)\right\rangle=e^{i \theta}\left|\left\langle\mathcal{O}_{i}(\infty)\right\rangle\right|\left(1-\frac{\xi_{2}^{2}}{2 \rho^{2}}+\ldots\right)
$$

to the tail of the condensate field or from the slope of the condensate in the vortex core.

$$
\left\langle\mathcal{O}_{i}(\rho)\right\rangle=e^{i \theta}\left|\left\langle\mathcal{O}_{i}(\infty)\right\rangle\right| \frac{\rho}{\xi_{1}}+\ldots
$$

As discussed in Appendix $\mathrm{C}$, in the large $\rho$ regime the differential equations may also be solved using shooting methods. The length scale in (29) may be determined with high accuracy. We will conventionally define the length scales as was done for solutions of the GP equation 2.1,

The boundary value of the derivative of the gauge field $A_{t}$ is identified with the boundary theory charge density $\rho_{q}$ (or the total particle number density in the superfluid language). It is seen that it can be fitted well to a function of the form

$$
\rho_{q}(\rho)=\rho_{q}(0)+\left(\rho_{q}(\rho)-\rho_{q}(0)\right)\left(1-\frac{\xi_{q}^{2}}{\rho^{2}}+\ldots\right)
$$

with a characteristic charge density coherence length $\xi_{q}$.

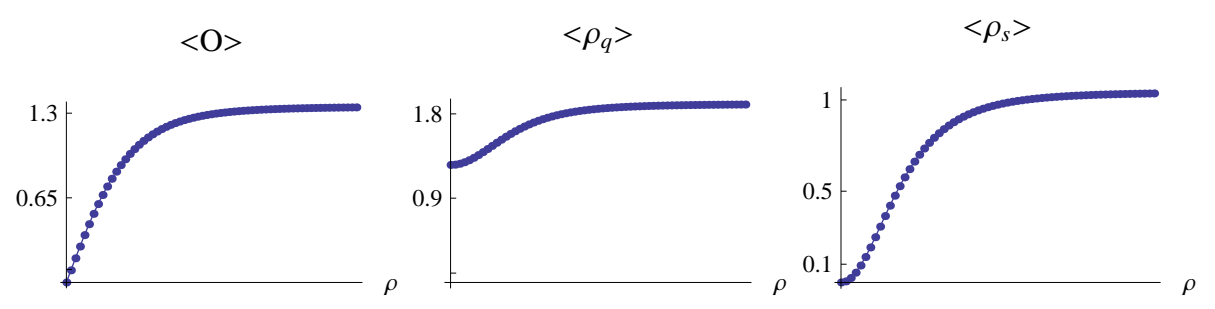

Figure 4: Typical radial profiles for expectation values.

The boundary value of the derivative of the angular gauge field $A_{\theta}$ can be identified with a current $j_{\theta}$ in the boundary theory. We will define the superfluid density $\rho_{s}$ through the relation

$$
j^{i}=\rho_{s} v_{s}^{i}
$$

Since $\chi(z=0)$ is the phase of the operator expectation value

$$
\left\langle\mathcal{O}_{i}\right\rangle=\tilde{R}_{i} e^{i \chi(z=0)}
$$




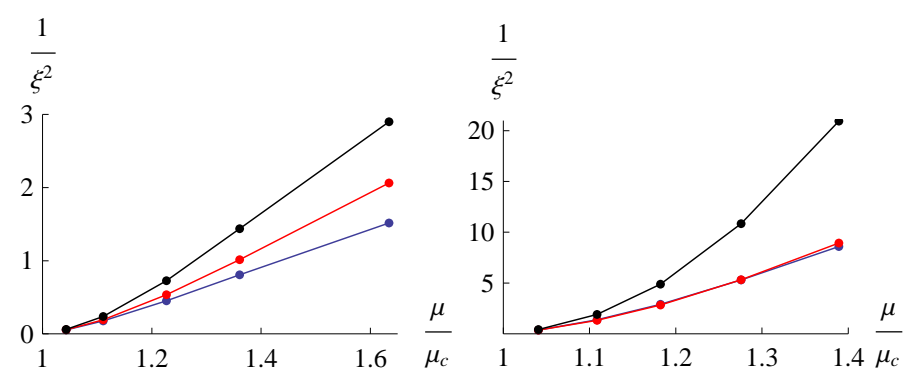

Figure 5: The length scales related to different operator expectation values. The different colors blue (up), black (middle) and red (down) correspond to the scales of the condensate, superfluid density and charge density, respectively. Left is for $\mathcal{O}_{1}$ and right is for $\mathcal{O}_{2}$.

we can identify the superfluid velocity as

$$
v_{s}^{i}=\frac{1}{\mu} \partial^{i} \chi(z=0)=\frac{1}{\mu} \frac{n \varepsilon^{i j} x^{j}}{\rho^{2}},
$$

where the normalization factor follows from relativistic symmetry [30] as we mentioned earlier. Now the superfluid density for the vortex can be identified as

$$
\rho_{s}=\frac{\mu \partial_{z} A_{\theta}(z=0)}{n} .
$$

We can identify a third scale by fitting the superfluid density as

$$
\rho_{s}(\rho)=\rho_{s}(\infty)\left(1-\frac{\xi_{s}^{2}}{\rho^{2}}+\ldots\right) .
$$

In Fig.(15), the different scales as determined from the asymptotic forms (29), (31) and (36) are graphed. As was found for holographic dark solitons, the length scales typically have different dependences on the chemical potential, indicating that there are independent scales governing the physics. There is one exception to this result, for the $\mathcal{O}_{2}$ superfluid, the condensate and charge density length scales coincide.

Another interesting quantity is the ratio of the condensate length scale determined from the core to the one determined from the tail $\xi_{1} / \xi_{2}$. It was noted in [17] that for a BCS superfluid, near zero temperature, these two length scales are very different. The basic idea behind this is that the physics at the vortex core is determined by the normal phase properties of the superfluid, so that the length scale is related to the microscopic Fermi momentum, $\xi_{1} \sim 1 / k_{F}$, while the length scale in the vortex tail is determined by the physics of the superfluid phase, where the relevant scale is the inverse gap $\xi_{2} \sim 1 / \Delta$. Thus, in weakly coupled BCS theory $\xi_{1} \ll \xi_{2}$. For a more tightly bound superfluid it was found in [17] that the two length scales coincide at 


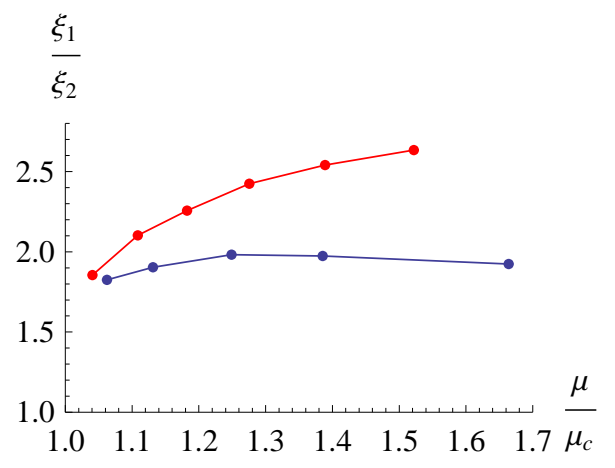

Figure 6: The ratio of the core length scale to the tail length scale of the condensate as a function of the chemical potential. $\mathcal{O}_{1}$ is blue and $\mathcal{O}_{2}$ is red.

the unitarity limit (infinite scattering length) and on the BEC side of the BCS-BEC crossover 5

Our results for the ratio $\xi_{1} / \xi_{2}$ in a holographic superfluid are shown in Figure 6. There are two features worth noting. First, for the $\mathcal{O}_{1}$ superfluid the ratio of tail to core length scales is consistent with a constant ratio at low temperatures (large $\mu$ ). As for the GP equation, we estimate the core length scale using the slope of the condensate at the origin. There is a relatively large uncertainty in the finding the slope in the core because the spatial gradients are large (especially at large $\mu$ ). The uncertainty in the length scales may be estimated as in [6]. We find that the uncertainty ranges from $1-10 \%$ with increasing chemical potential. For the $\mathcal{O}_{2}$ superfluid the ratio of length scales clearly depends on the chemical potential, consistent with there being two distinct length scales in the vortex's core and tail.

The second feature we note is that the ratio of the length scales is larger for $\mathcal{O}_{2}$ than for $\mathcal{O}_{1}$. Given that other quantities (including the density deplitions and the critical velocities) indicated that $\mathcal{O}_{2}$ might be a BCS-like superfluid. This is a surprising feature. In a speculative vein, if we assume that the $\mathcal{O}_{2}$ liquid is a fermionic superfluid, we would interpret the absence of a small core length scale as suggesting a vanishing Fermi momentum. This is also supported by the fact that we do not see any Friedel oscillations in our solutions, which would have a wavelength proportional to $k_{F}$ [16, 17]. One possibility is that, there are a large number of charged fermion species $N$ contributing to the condensate. In this way, one could put an infinite charge to the ground state without having to occupy higher energy states (assuming the charge density is finite), which would mean that the Fermi surface, and thus the Fermi momentum would scale to zero as $N \rightarrow \infty$.

\footnotetext{
${ }^{5}$ We found in section 2.1 that the ratio of the two length scales is not one, but this is not really to be expected, but rather both of them are proportional to the same length scale $\xi=1 / \sqrt{\mu^{2}-V}$.
} 


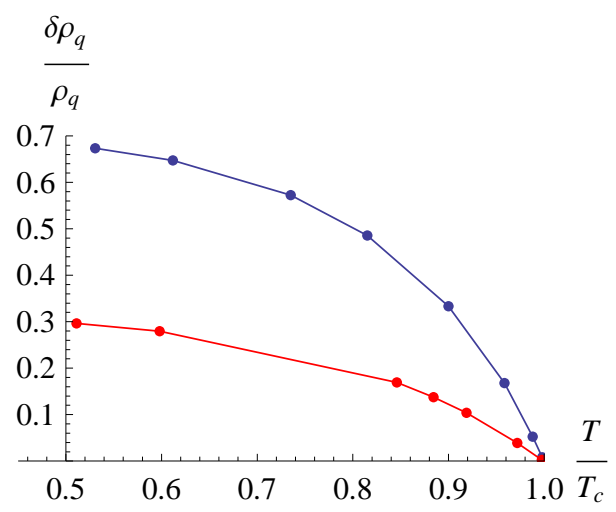

Figure 7: The density depletion fraction as a function of temperature. The upper curve is $\mathcal{O}_{1}$ (blue) while the lower curve is $\mathcal{O}_{2}$ (red).

\section{Density depletion}

An interesting observable in the vortex solutions is the charge density profile. It carries non-trivial information about the fraction of the charged matter in the condensate. Near $T=T_{c}$ the density depletion in the core of the vortex is very small, which suggests that the part of charged matter in the condensate is small compared to the total charge density. This is found for both condensing operators $\mathcal{O}_{1}$ and $\mathcal{O}_{2}$.

A pronounced difference between $\mathcal{O}_{1}$ and $\mathcal{O}_{2}$ becomes clear when the temperature is lowered. For $\mathcal{O}_{2}$ the density depletion fraction seems to be saturating at $40 \%$ while for $\mathcal{O}_{1}$ it is likely to grow near $100 \%$. This same basic pattern was also observed for holographic dark solitons [5, 6]. Comparing the density depletions to those obtained in a non-relativistic setting [17] suggests that one may identify $\mathcal{O}_{2}$ as a BCS type superfluid and $\mathcal{O}_{1}$ as a BEC type superfluid.

\section{Critical velocity}

An interesting quantity in the context of superfluids is the critical superfluid velocity, above which the superfluidity of the system is destroyed and the flow of the fluid starts to dissipate.

\subsection{Landau criterion}

At low temperature the critical superfluid velocity can be estimated by the Landau criterion, which goes as follows. Consider the superfluid moving in a container with a velocity $\mathbf{v}$. Dissipation occurs when the wall of the container or some other defect 

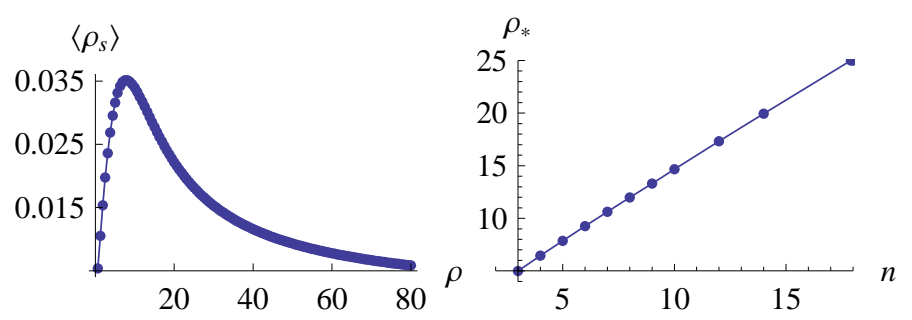

Figure 8: The left figure shows a typical profile for the supercurrent in a vortex. The right figure shows the critical radius for different values of $n$ and it seems to be a linear function.

starts to excite quasiparticles in the fluid 6 . Let us consider a quasiparticle with energy $\varepsilon_{p}$ and spatial momentum p. Obviously these together form the momentum four vector. As we boost back to the rest frame of the container, the time component of the four momentum transforms into

$$
\varepsilon^{\prime}=\frac{\varepsilon_{p}+\mathbf{p} \cdot \mathbf{v}}{\sqrt{1-v^{2}}} .
$$

Whenever the energy of the quasiparticle $\varepsilon^{\prime}$ is negative, as measured from the rest frame of the container, it becomes energetically favorable to create such excitations. The expression (37) is minimized when $\mathbf{p}$ and $\mathbf{v}$ are antiparallel. This gives us the Landau critical velocity

$$
v_{c r i t}=\min _{p} \frac{\varepsilon_{p}}{|\mathbf{p}|},
$$

where the minimum is taken over all possible quasiparticle excitations. This shows that it is not necessary for the quasiparticle spectrum to be gapped, but there can be excitations with linear dispersion relation for small momenta.

\subsection{Determination of the critical velocity from the vortices}

We can determine a critical velocity from the vortices as follows. We begin by asking a simple question: Why does the radius of a vortex increase as the winding number is increased? The simplest physical reason is that the velocity of the superfluid flow around the vortex increases as $v \sim n / \rho$ and at some radius $\rho_{*}$ the superfluid velocity gets larger than the critical velocity and inside that radius the condensate vanishes since it is no longer energetically favorable.

Within our numerical solutions we can easily test this idea, by plotting the critical radius $\rho_{*}(n)$ and seeing whether it behaves as a linear function of the winding $n$, as should be if the idea of critical velocity is the correct physical reason for the grow of the

\footnotetext{
${ }^{6}$ For the dissipation one does not need a container, it is enough to have relative motion between superfluid and normal components.
} 


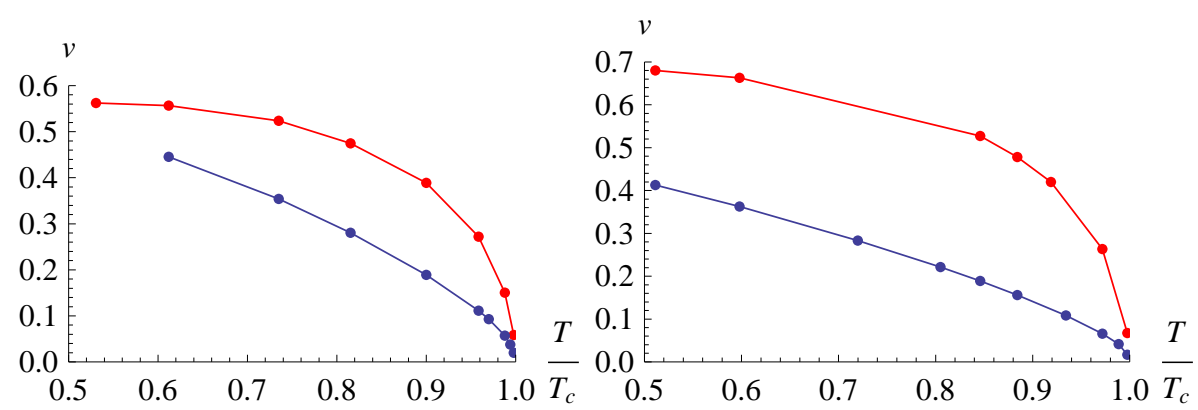

Figure 9: The upper curve is the sound velocity and the lower curve is the critical velocity as a function of the temperature as determined from the vortices. $\mathcal{O}_{1}$ is left and $\mathcal{O}_{2}$ is right.

vortices. Following ideas from [17], we define the critical radius to be the point where the current $j^{i}(\rho)$ reaches its maximum. A typical profile of the supercurrent $j_{s}(\rho)$ for a vortex is shown in Fig.(8). Inside the a critical radius, where the supercurrent reaches its maximum value, the superfluid density starts to decrease causing the supercurrent to decrease. The critical radius as a function of the winding number, $\rho_{*}(n)$ seems to be indeed a linear function of $n$ as shown in Fig.(8).

By generating vortex solutions with a high winding number $n=20$, for different values of the chemical potential (or equivalently, for different values of the temperature) we can see the behavior of the critical velocity as a function of the chemical potential. The results are shown in Fig.(9) for different condensing operators $\mathcal{O}_{1}$ and $\mathrm{O}_{2}$.

Near the critical temperature the functional form of the critical velocity fits well with a square root behavior

$$
v_{c} \approx v_{0} \sqrt{1-\frac{T}{T_{c}}}
$$

This is the same functional form as was found in [3]. It is interesting to compare the critical velocity determined by the vortices to the Landau critical velocity. For bosonic superfluids and tightly bound fermionic superfluids, the quasiparticle determining the Landau critical velocity is a collective mode (sound mode) [33]. A vortex's critical velocity depends on the type of superfluid. For a weakly coupled fermionic superfluid it is the breaking of Cooper pairs that determines the critical velocity, while for a bosonic superfluid a sound mode sets the relevant scale. In [34] it is found that the second sound is the smallest (known) sound velocity. Thus, it should determine the Landau critical velocity for a bosonic superfluid. We can use this to probe the superfluid type. In Fig.(9), the critical velocities are plotted with the corresponding sound velocities.

At low temperatures (large $\mu$ ), for the $\mathcal{O}_{1}$ superfluid, the critical velocity is closer 
to the sound velocity than for the $\mathcal{O}_{2}$ superfluid. This indicates that the vortex critical velocity is more likely to be set by a sound mode for the $\mathcal{O}_{1}$ than for $\mathcal{O}_{2}$.

\section{Free energy and a puzzle with the BKT argu- ment}

Next, we will show that the vortex solutions have a logarithmically divergent free energy as is usual in superfluids (for a related calculation see [8]). According to the AdS/CFT dictionary the free energy of the boundary QFT is identified with the Euclidean on shell action in the bulk AdS. First we will evaluate the Lorenzian onshell action on the vortex solution, with a cutoff $z=\epsilon$ at the AdS boundary and an infrared cutoff at the radial position $\rho=R_{c}$.

$$
\begin{aligned}
S_{A d S} & =\int d t \int_{\epsilon}^{1} d z \int_{0}^{2 \pi} d \theta \int_{0}^{R_{c}} \rho d \rho\left(-\frac{f}{z^{2}}\left|\partial_{z} \Psi\right|^{2}-\frac{1}{z^{2}}\left|\partial_{\rho} \Psi\right|^{2}\right. \\
& +\frac{1}{f z^{2}} A_{t}^{2}|\Psi|^{2}-\frac{m^{2}}{z^{4}}|\Psi|^{2}-\frac{1}{2 \rho^{2}}\left(\partial_{\rho} A_{\theta}\right)^{2}+\frac{1}{2}\left(\partial_{z} A_{t}\right)^{2} \\
& \left.+\frac{1}{2 f}\left(\partial_{\rho} A_{t}\right)^{2}-\frac{f}{2 \rho^{2}}\left(\partial_{z} A_{\theta}\right)^{2}-\frac{1}{z^{2} \rho^{2}}\left|\partial_{\theta} \Psi-i A_{\theta} \Psi\right|^{2}\right)
\end{aligned}
$$

After substracting a counterterm $S_{c t}=\int d^{3} x|\Psi|^{2} / \epsilon^{3}$ the Lagrangian density is finite everywhere for the vortex solutions, but the action diverges due to integral over $\rho$. Thus, leading terms in the free energy are the diverging ones as $R_{c} \rightarrow \infty$. We will concentrate here on these terms. Because the diverging terms are a large $\rho$ effect, they may be captured using the asymptotic expansion in Appendix C]

$$
\tilde{R}=\tilde{R}^{0}(z)+\frac{\delta \tilde{R}(z)}{\rho^{2}}, \quad A_{t}=A_{t}^{0}(z)+\frac{\delta A_{t}(z)}{\rho^{2}}, \quad A_{\theta}=A_{\theta}^{0}(z)+\frac{\delta A_{\theta}(z)}{\rho^{2}}
$$

At leading order in this expansion the Lagrangian density is simply $\mathcal{L}\left(\tilde{R}=\tilde{R}^{0}, A_{t}=\right.$ $\left.A_{t}^{0}, A_{\theta}=0\right)$. This gives rise to an extensive term in the free energy diverging as $R_{c}^{2}$, which is exactly the free energy of the translationally invariant symmetry breaking state. At subleading order there are logarithmic divergences in the free energy. These have two sources, the two last terms in (40) are log divergent when evaluated on $A_{\theta}^{0}(z)$, while the power law correction to $A_{\theta}$ in (41) gives finite subleading terms. Another source of possible log divergences are the first order corrections to $\tilde{R}$ and $A_{t}$ in (41). It is easy to see that these terms have to vanish, since they give rise to terms that are proportional to the asymptotic equations of motion (173), schematically

$$
\int d^{4} x\left(\frac{\delta S}{\delta A_{t}} \frac{\delta A_{t}(z)}{\rho^{2}}+\frac{\delta S}{\delta \tilde{R}} \frac{\delta \tilde{R}(z)}{\rho^{2}}\right)
$$




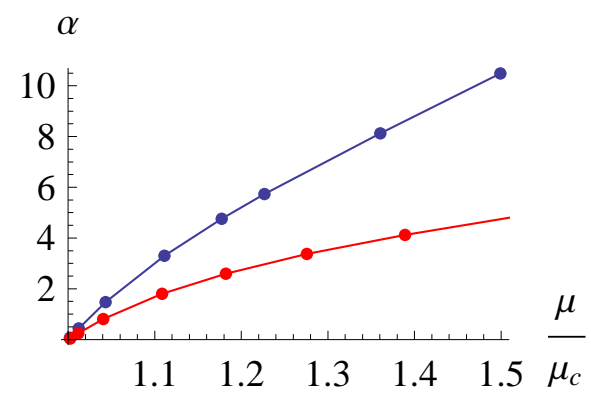

Figure 10: Free-energy coefficients as functions of the chemical potential. Blue (up) is $\mathcal{O}_{1}$ and red (down) is $\mathcal{O}_{2}$.

and to boundary terms $\delta A(0) \partial_{z} A_{t}^{0}(0)$ and $\delta \tilde{R}(0) \partial_{z} \tilde{R}(0)$, which both are vanishing. Thus, we see that the difference between action evaluated on the translationally invariant symmetry breaking solution and the vortex is given by

$$
\Delta S=-2 \pi \log \left(\frac{R_{c}}{\xi}\right) \int d t d z\left[\frac{1}{2}\left(\tilde{R}^{0}\right)^{2}\left(n-A_{\theta}^{0}\right)^{2}+\frac{f}{2}\left(\partial_{z} A_{\theta}^{0}\right)^{2}\right],
$$

where we have neglected terms that are finite in the limit $\rho \rightarrow \infty$. By using the equations of motion of the $A_{\theta}$ field we see that the integrand in (43) becomes simply $n \partial_{z}\left(f \partial_{z} A_{\theta}\right)$. By continuing the action to Euclidean time with period $\beta=1 / T$ we end up with the free energy difference

$$
\Delta \Omega=\alpha \log \left(\frac{R_{c}}{\xi}\right),
$$

where $\alpha=\left.\pi n\left(\partial_{z} A_{\theta}\right)\right|_{z=0}$. The coefficient $\alpha$ is shown in figure 10 as a function of the chemical potential. In the section 5 we showed that the superfluid density for the vortex solution is simply $\rho_{s}=\mu\left(\partial_{z} A_{\theta}\right) / n$. Thus, we see that the vortex free energy is given by

$$
\Delta \Omega=\frac{\pi \rho_{s}(\infty) n^{2}}{\mu} \log \left(\frac{R_{c}}{\xi}\right),
$$

where $\xi$ is a length scale which measures the vortex core size. The vortex free energy agrees exactly with the one determined from symmetry arguments in section 2, as it should. Furthermore, $\alpha / 2 \pi$ is the coupling constant in front of the Goldstone effective action for the holographic model.

There is a slight puzzle involved with the free energy as it vanishes at $T=T_{c}$. Even though we have calculated the vortex free energy, we are missing a piece involving the vortex entropy, since we have fixed the center of the vortex. The entropy of a single vortex is simply the number of possible vortex states. Since the vortex occupies an area of order $\xi^{2}$, the number of possible vortex positions is $R_{c}^{2} / \xi^{2}$ and thus, the 
entropy is $S=\log \left(R_{c}^{2} / \xi^{2}\right) 7$ The free energy of a single vortex is

$$
F=E-T S=(\alpha-2 T) \log \left(\frac{R_{c}}{\xi}\right)
$$

This suggests that at some temperature below the critical temperature of the phase transition described in [1, it becomes entropically favorable for a Berezinskii-KosterlitzThouless (BKT) transition. In such transitions it is entropically favorable for vortex/antivortex pairs to deconfine, destroying the superfluid state. A potential pitfall of the above argument is that the semi-classical gravity approximation involves a large- $N$ limit. There is the possibility of an order of limits issue when taking both $N$ and $q \rightarrow \infty$. It is possible that factors of $N$ enter into the ratio $E / T$ but do not effect the entropy $S 8$ In this case there would be an order of limits issue, which might drive the BKT transition temperature to the superfluid transition temperature. To clarify this issue it may be necessary to include $1 / N$ corrections. We will save a more detailed study for later work. It would also be interesting to see where the potential BKT transition is placed in the phase diagrams of [35].

\section{Discussion}

In this work we have studied vortex configurations permitted in holographic superfluids. These vortex solutions are characterized by a depletion of the condensate and charge density in the vortex core and the quantization of the circulation around the vortex. In the bulk these solutions correspond to vortex configurations in an Einstein-Maxwell-Higgs system, which have vanishing boundary values for the spatial components of the vector field. Bulk fluxes do not become boundary gauge fields, but instead map into the charge and superfluid densities in the boundary theory.

We explored features of the vortices as functions of the chemical potential and type of condensing operator. We have used the fact that vortex configurations are not small deviations from the homogeneous condensate to probe both long and short distance features of holographic superfluids. There has been much work interpreting the system of [2] as a superconductor. This may be appropriate for features which do not require a dynamical gauge field (such as conductivity calculations and the superconducting gap). However, for other features, such as the Meissner effect, a dynamical gauge field and Maxwell's equations are required. Then, vortices act as sources for the gauge field and require magnetic fluxes. However, the existence of vortex solutions even in the absence of a magnetic field on the boundary indicates

\footnotetext{
${ }^{7}$ Rather than appeal to this heuristic derivation of the entropy one can see the same term arising when one path integrates the moduli associated with translating the vortex core in the $\rho-\theta$ plane.

${ }^{8} S$ is determined by the metric on moduli space and its leading contribution is independent of coupling constants in the gravitational theory
} 
that these systems may more likely be a superfluid or a thin film superconducting states.

The key feature of a thin superconducting film of thickness $d$ is that the magnetic fields, as they live in 3 spatial dimensions, spread out in space outside the film and thus make the penetration depth enhanced into $\lambda_{\text {eff }}=\lambda^{2} / d \gg \lambda$. For a thin film $\lambda_{\text {eff }}$ can be really large (for $d \approx 10-100$ Angstroms, $\lambda_{\text {eff }} \approx \mathcal{O}(1 \mathrm{~cm})$ ), and the dynamics of vortices in the system is equivalent to that of a superfluid. It becomes possible to see a BKT phase transition in a superconductor. This enhancement was noted in [36] and for a nice review see [37]. Now for a single vortex that has a magnetic flux of a one flux quantum $\Phi_{0}$, the magnetic field is spread on a large area $A \sim \lambda_{\text {eff }}^{2}$. Thus the magnitude of the magnetic field inside the vortex core is of the order $B \sim \Phi_{0} / A, 9$ which is practically zero. Thus, when modeling vortices in a thin superconducting film it is a good approximation to set the local magnetic field to zero.

The specific system studied here is a relativistic superfluid, but the features of nonrelativistic superfluid vortices are a useful guide for interpreting our results. Specifically, we find that vortex configurations have distinct length scales characterizing the variations of the condensate, charge density, and superfluid density. Each of these length scales is characterized by a $1 / \sqrt{\mu / \mu_{c}-1}$ near the critical point. However, each of these scales generically has a different dependence on the chemical potential for larger $\mu$ as is seen in Fig.(5).

As with holographic dark solitons [5,6] the behavior of the charge density depletion fraction of the vortex core is very different for the $\left\langle\mathcal{O}_{1}\right\rangle$ and $\left\langle\mathcal{O}_{2}\right\rangle$ condensates. For both dark solitons and vortices, the $\left\langle\mathcal{O}_{1}\right\rangle$ condensate has near $100 \%$ charge depletion in the core for low temperature (large $\mu$ ). For $\left\langle\mathcal{O}_{2}\right\rangle$ the core density depletion is much more modest, near $40 \%$. This is consistent with the picture that the $\left\langle\mathcal{O}_{1}\right\rangle$ condensate is BEC-like, comprised of a point-like boson. Similarly, this is suggestive that the $\left\langle\mathcal{O}_{2}\right\rangle$ condensate is more BCS-like, comprised of non-local Cooper pairs.

In [17] the impact that varying the type of superfluid across the BEC-BCS crossover has on vortices was explored. In that non-relativistic system, it was found that for BEC superfluids there is a single length scale characterizing the variations of the condensate. However, for a BCS superfluid the condensate profile was characterized by two length scales. In the vortex's tail the characteristic length scale is set by the size of the gap. Near the core, as the system locally approaches the normal phase, $k_{F}$ determines the characteristic scale. In Fig (6) we see some evidence that similar things happen for holographic superfluids. Specifically we see that there is really one length scale characterizing both the core and tail region for an $<\mathcal{O}_{1}>$ type condensate. For $<\mathcal{O}_{2}>$ the core and tail are characterized by two distinct scales. However,

\footnotetext{
${ }^{9} \mathrm{~A}$ similar scaling of the magnetic field with the area was used in $[8$, but there the area $A$ is kept finite and thus, the external magnetic field is nonzero.
} 
the picture is not quite as clean as one might have hoped. In the non-relativistic system, the core length scale is smaller than for the tail. In the holographic $<\mathcal{O}_{2}>$ superfluid we find the opposite. If one accepts the basic picture that that the two types of holographic superfluids are of BEC and BCS types, on might view the core length scale as giving information about the Fermi surface (in the large $N$ limit). Specifically, we would conclude that the Fermi momenta is vanishing in the large $N$ limit. The vanishing of $k_{f}$ is consistent with the absence of Friedel oscillations in vortices and dark solitons [5, 6]. For other recent discussions of Fermi surfaces in holographic quantum liquids see [38-40]. It would be interesting to compare their results to the behavior we see in the vortex cores.

We also study the manner in which vortices allow one to estimate the critical velocity of the superfluid. Heuristically, this is due to the fact that the superfluid's local velocity increases beyond its critical velocity as one approaches the core. We can then estimate the critical velocity by the radius where the superfluid density starts to drop off. At low temperatures the Landau criterion sets an upper bound for the critical velocity of the superfluid in terms of the lowest sound mode. The fact that the $\mathcal{O}_{1}$ superfluid's critical velocity comes closest to saturating the Landau criterion indicates that it is more likely to be a BEC-like superfluid (whose critical velocity is set by a sound mode).

In addition to studying the vortices scales of variation, we confirmed that their energy cost over the homogeneous solution diverges logrithmically. While this is generically for any $2+1$ dimensional vortex, the holographic reason is that the exterior of an AdS-Schwarzschild black hole effectively acts as a finite box in the radial direction. This is a nice confirmation that the bulk vortices give rise to superfluid vortices in the boundary theory as opposed to superconductor vortices. On general grounds one expects vortices in a superconductor to be finite energy excitations.

In addition it appears that one can have a BKT transition at temperatures below the temperature of spontaneous symmetry breaking. To definitively resolve this issue on may need to include $1 / N$ effects as well as gravitational backreaction. As in [5, 6], the absence of gravitational back reaction limits our ability to take the zero temperature limit of vortex configurations. Including gravitational backreaction would allow us to see many features unobstructed by thermal fluctuations. In addition, the backreacted geometry would present a very novel example of black hole hair. For recent work on black holes with inhomogeneous hair see [41].

Throughout this paper and in studies of dark solitons we have repeatedly seen features suggesting that the the choice of quantization in $A d S_{4}$ corresponds to two types of superfluids. It would be worthwhile to probe this feature more closely. Ideally one would like to understand if there is any holographic analog of the "unitarity" limit in the BEC-BCS crossover. One way to probe this physics might be to vary the $m^{2}$ parameter in the bulk Lagrangian (studies in this direction were performed in [21,42, 43]). If we associate the operator scaling dimension with the type of superfluidity, it 
is interesting to speculate that the unitarity limit would correspond to saturating the BF bound, $m^{2}=-9 / 4$, in $A d S_{4}$. One way that one might try to see this would be to study the way that the critical velocity varies with $m^{2}$. In [17] it was shown that the critical velocity was not a monotonic function across the BEC-BCS crossover. The peak value occurrs in the unitarity limit. Since the system is relativistic one might be able to go all the way from BCS to BEC and to relativistic Bose-Einstein condensation (RBEC) [44.

Finally, it would be very interesting to explore all of these questions in a setting more appropriate for laboratory experiments. Specifically we anticipate all of these ideas have analogs in non-relativistic gauge-gravity duals. It would be very interesting to study vortices in the backgrounds described in [45,48]

Acknowledgements We would like to thank Clifford Johnson, Rob Leigh, Makoto Natsuume, and Kari Rummukainen for useful comments. V.K. and E.K-V. have been supported in part by the Academy of Finland grant number 1127482. E.K-V. and S.N. thank the Aspen Center for Physics for their hospitality during the early stages of this work.

\section{A Gauge fixing}

In this appendix we will outline gauge fixing necessary to obtain the equations in section 3. Because vortex solutions are cylindrically symmetric in the $(\rho, \theta)$ plane, we can take all gauge invariant quantities independent of the angle $\theta$. Since we are interested in static solutions, we will also take all the fields to be independent of the time coordinate 10 Choosing the gauge, $A_{z}=0$, and using $\partial_{\theta} R=0$ (since $R$ is gauge

\footnotetext{
${ }^{10}$ More general condition would have been to take all the gauge invariant quantities independent of $t$, but this leads to trouble discussed in [1]
} 
invariant) equations (19), (20) and (21) become

$$
\begin{aligned}
0 & =z \partial_{z}\left(\frac{f}{z^{2}} \partial_{z} R\right)+\frac{m^{2}}{z^{2}}\left(\frac{R}{z}\right)+\frac{1}{\rho} \partial_{\rho}\left(\rho \partial_{\rho}\left(\frac{R}{z}\right)\right) \\
& -\left(\frac{R}{z}\right)\left(-\frac{1}{f} A_{t}^{2}+f\left(\partial_{z} \chi\right)^{2}+\frac{\left(A_{\theta}-\partial_{\theta} \chi\right)^{2}}{\rho^{2}}+\left(A_{\rho}-\partial_{\rho} \chi\right)^{2}\right) \\
0 & =\frac{1}{\rho} \partial_{\rho}\left(\rho \partial_{z} A_{\rho}\right)+\frac{1}{\rho^{2}} \partial_{\theta} \partial_{z} A_{\theta}-\left(\frac{R}{z}\right)^{2} \partial_{z} \chi \\
0 & =f \partial_{z}^{2} A_{t}+\frac{1}{\rho} \partial_{\rho}\left(\rho \partial_{\rho} A_{t}\right)+\frac{1}{\rho^{2}} \partial_{\theta}^{2} A_{t}-\left(\frac{R}{z}\right)^{2} A_{t} \\
0 & =\partial_{z}\left(f \partial_{z} A_{\rho}\right)+\frac{f}{\rho^{2}} \partial_{\theta}^{2} A_{\rho}-\frac{f}{\rho^{2}} \partial_{\theta} \partial_{z} A_{\theta}-\left(\frac{R}{z}\right)^{2}\left(A_{\rho}-\partial_{\rho} \chi\right) \\
0 & =\partial_{z}\left(f \partial_{z} A_{\theta}\right)+\rho \partial_{\rho}\left(\frac{1}{\rho} \partial_{\rho} A_{\theta}\right)-\rho \partial_{\rho}\left(\frac{1}{\rho} \partial_{\theta} A_{\rho}\right)-\left(\frac{R}{z}\right)^{2}\left(A_{\theta}-\partial_{\theta} \chi\right) \\
0 & =\partial_{z}\left(f\left(\frac{R}{z}\right)^{2} \partial_{z} \chi\right)-\frac{1}{\rho} \partial_{\rho}\left(\rho\left(\frac{R}{z}\right)^{2}\left(A_{\rho}-\partial_{\rho} \chi\right)\right) \\
& -\frac{1}{\rho^{2}} \partial_{\theta}\left(\left(\frac{R}{z}\right)\left(A_{\theta}-\partial_{\theta} \chi\right)\right) .
\end{aligned}
$$

Furthermore, to model a vortex we want the radial current to vanish, that is, $A_{\rho}-$ $\partial_{\rho} \chi=0$.

We may use the residual gauge invariance and the requirements of regularity along the horizon and in the vortex core to simplify the remaining equations. Using $\partial_{\theta}\left(\partial_{\theta} \chi-A_{\theta}\right)=0$, equation (52) implies

$$
\partial_{z}\left(\left(\frac{R}{z}\right)^{2} f(z) \partial_{z} \chi\right)=0
$$

Which has the solution

$$
\partial_{z} \chi=\frac{z^{2} C(\rho)}{R^{2} f(z)} .
$$

We can note that $C$ is independent of $\theta$ since $0=\partial_{\theta}\left(\partial_{z} \chi-A_{z}\right)=\partial_{\theta} \partial_{z} \chi$. Regularity of $\chi$ at the horizon forces us to set $C(\rho)=0$.

Cylindrical symmetry implies that $\partial_{\theta} F_{\rho \theta}=0$ and $\partial_{\theta} F_{\theta z}=0$. Substituting these in (50), gives

$$
\partial_{z}\left(f \partial_{z} A_{\rho}\right)=0
$$

which has a solutions $\partial_{z} A_{\rho}=\frac{D(\rho, \theta)}{f(z)}$. Again, regularity at the horizon requires $D=0$. This means that $A_{\rho}=A_{\rho}(\theta, \rho)$. Thus, by a gauge transformation we can set $A_{\rho}=$ $0=\partial_{\rho} \chi$

The symmetry also imposes $\partial_{\theta} F_{z \theta}=\partial_{\theta} \partial_{z} A_{\theta}=0$, This has the general solution $A_{\theta}=A_{\theta}^{(1)}(\theta, \rho)+A_{\theta}^{(2)}(z, \rho)$. From $\partial_{\theta}\left(\partial_{\theta} \chi+A_{\theta}\right)=0$ and $\partial_{\rho} \chi=0$ we can conclude that $A_{\theta}^{(1)}$ is independent of $\rho$ and can be thus absorbed into $\chi$ with a gauge transformation. Thus $A_{\theta}=A_{\theta}(z, \rho)$. 
Similarly, cylindrical symmetry also requires $0=\partial_{\theta}\left(\partial_{\theta} \chi+A_{\theta}\right)=\partial_{\theta}^{2} \chi$, which implies that $\chi=\alpha+\beta \theta$ for $\alpha$ and $\beta$ constants. In order for $\Psi$ to be a single valued function $\beta$ has to be an integer $n \in \mathbb{Z}$, which is the winding number of the vortex. We will also set $\alpha=0$ as a part of the gauge choice. Note that the field $\chi(z, x)$ is completely determined by the equations of motion, and thus, cannot be thought of as a non-normalizable mode 11 The final simplification is due to the observation that $A_{t}-\partial_{0} \chi=A_{t}$ is gauge invariant, and therefore is independent of $\theta$

Taking advantage of the cylindrical symmetry, the equations may be written

$$
\begin{aligned}
0 & =z \partial_{z}\left(\frac{f}{z^{2}} \partial_{z} R\right)+\frac{m^{2}}{z^{2}}\left(\frac{R}{z}\right)+\frac{1}{\rho} \partial_{\rho}\left(\rho \partial_{\rho}\left(\frac{R}{z}\right)\right) \\
& -\left(\frac{R}{z}\right)\left(-\frac{1}{f} A_{t}^{2}+\frac{\left(A_{\theta}-n\right)^{2}}{\rho^{2}}\right) \\
0 & =f \partial_{z}^{2} A_{t}+\frac{1}{\rho} \partial_{\rho}\left(\rho \partial_{\rho} A_{t}\right)-\left(\frac{R}{z}\right)^{2} A_{t} \\
0 & =\partial_{z}\left(f \partial_{z} A_{\theta}\right)+\rho \partial_{\rho}\left(\frac{1}{\rho} \partial_{\rho} A_{\theta}\right)-\left(\frac{R}{z}\right)^{2}\left(A_{\theta}-n\right) .
\end{aligned}
$$

After defining $\tilde{R}=R / z$, these equations are equivalent to (22/24).

\section{B Boundary conditions for the $\chi$ field}

According to the AdS/CFT dictionary the expectation value of the order parameter field is given by

$$
\left\langle\mathcal{O}_{i}\right\rangle \sim e^{i \chi(0, x)} R_{(i)}(0, x) .
$$

Thus, $\chi(0, x)$ should be a quantity determined by the state one is looking at. Therefore one should not be able to choose the value of $\chi(0, x)$ as a boundary condition. We will show in this appendix that this is indeed the case for a general time independent solution of the equations of motion. The equations of motion for $\chi$ in terms of the $\tilde{R}$ field are in the gauge $A_{z}=0$ given by

$$
\partial_{z}\left(f \tilde{R}^{2} \partial_{z} \chi\right)+\partial_{i}\left(\tilde{R}^{2}\left(\partial_{i} \chi-A_{i}\right)\right)=0 .
$$

Since (60) is elliptic, boundary conditions should be provided at every spatial boundary. Since at the horizon (60) becomes parabolic, it provides an effective boundary condition at the horizon (this is usually referred as a regularity condition). Next we will show that the other boundary condition at the AdS boundary is fixed by the equations of motion for the $\mathcal{O}_{2}$ condensate and by a "regularity" condition for the $\mathcal{O}_{1}$ condensate.

\footnotetext{
${ }^{11}$ In Appendix B we show that for a general solution of the field equations (19), (20) and (21), the phase field $\chi$ is completely determined by the equations of motion and there are no freedom to choose boundary conditions for $\chi$ at the AdS boundary.
} 
We know the asymptotic expansion for the fields, $\tilde{R}=R_{(1)}+z R_{(2)}+\ldots$ and $A_{\mu}=A_{\mu}^{(0)}+z A_{\mu}^{(1)}+\ldots$. By regularity the expansion for $\chi$ should come in positive integer powers $\chi=\chi_{(0)}+z \chi_{(1)}+\ldots$. Plugging these expansions into (60) gives the following equation up to second order in the powers of $z$

$$
\begin{aligned}
& 2 R_{(1)} R_{(2)} \chi_{(1)}+2 R_{(1)}^{2} \chi_{(2)}+\partial_{i}\left(R_{(1)}^{2}\left(\partial_{i} \chi_{(0)}-A_{i}^{(0)}\right)\right)=0 . \\
& 6 \chi_{(3)} R_{(1)}^{2}+2\left(R_{(2)}^{2}+2 R_{(1)} R_{(3)}\right) \chi_{(1)}+8 R_{(1)} R_{(2)} \chi_{(2)} \\
& +2 \partial_{i}\left(R_{(1)} R_{(2)}\left(\partial_{i} \chi_{(0)}-A_{i}^{(0)}\right)\right)+\partial_{i}\left(R_{(1)}^{2}\left(\partial_{i} \chi_{(1)}-A_{i}^{(1)}\right)\right)=0 .
\end{aligned}
$$

For the $\mathcal{O}_{2}$ condensate we have $R_{(1)}(x)=0$ and thus (61) is trivially solved, while (62) simplifies into $\chi_{(1)}=0$. This means that for $\mathcal{O}_{2}$, the equation of motion forces the Neumann boundary condition

$$
\partial_{z} \chi(z=0)=0
$$

and thus there is no more freedom to give boundary conditions to the $\chi$ field at the AdS boundary.

For the case of the $\mathcal{O}_{1}$ condensate we are not able to derive such a boundary condition from the equations of motion. Rather, we will use a physical argument, which sounds quite sensible. We require that the flux of the electric $U(1)$ current into the $z$ direction vanishes as we approach the AdS boundary, since literally the space ends there. This can be thought of as requiring the total charge in the system to be conserved 12 So we require

$$
\lim _{z_{0} \rightarrow 0} \int_{z=z_{0}} d^{2} x \sqrt{g^{(2)}} J^{z}=0
$$

The bulk current is given by the expression $J^{z}=\frac{-i}{2} \sqrt{-g} g^{z z}\left(\Psi^{*} \partial_{z} \Psi-\Psi \partial_{z} \Psi^{*}-\right.$ $\left.2 i A_{z}|\Psi|^{2}\right)=f \tilde{R}^{2} \partial_{z} \chi$. Now (64) implies that

$$
\lim _{z_{0} \rightarrow 0} \int_{z=z_{0}} d^{2} x \frac{f(z)}{z^{2}} \tilde{R}^{2} \partial_{z} \chi=0 .
$$

This can be true only if $\partial_{z} \chi(z=0)=0$.

\section{Large $\rho$ behavior}

In this appendix we outline an alternate approach to determine the large radius behavior of vortex solutions. The main observation is that for large $\rho$ the partial differential equations may be reduced to a set of coupled ordinary differential equations, which may be solve using Mathematica's NDSolve routine.

\footnotetext{
${ }^{12}$ A similar argument was used in [49] for the energy momentum tensor and conservation of the total energy, in order to derive the BF bound.
} 
In more detail, the gauge fixed equations of motion are:

$$
\begin{aligned}
& \partial_{z}\left(f \partial_{z} \tilde{R}\right)+\frac{1}{\rho} \partial_{\rho}\left(\rho \partial_{\rho} \tilde{R}\right)-\left(z+\frac{\left(A_{\theta}-n\right)^{2}}{\rho^{2}}-\frac{A_{t}^{2}}{f}\right) \tilde{R}=0 \\
& f \partial_{z}^{2} A_{t}+\frac{1}{\rho} \partial_{\rho}\left(\rho \partial_{\rho} A_{t}\right)-\tilde{R}^{2} A_{t}=0 \\
& \partial_{z}\left(f \partial_{z} A_{\theta}\right)+\rho \partial_{\rho}\left(\frac{1}{\rho} \partial_{\rho} A_{\theta}\right)-\tilde{R}^{2}\left(A_{\theta}-n\right)=0 .
\end{aligned}
$$

If we assume that that all of the fields become spatially homogeneous for large $\rho$, we see that the $\tilde{R}$ and $A_{t}$ reduce to the equations solved by [2]. We can then develop an expansion around this solution,

$$
\tilde{R}=\tilde{R}^{0}+\frac{\delta \tilde{R}(z)}{\rho^{2}}+\ldots, \quad A_{t}=A_{t}^{0}+\frac{\delta A_{t}(z)}{\rho^{2}}+\ldots, \quad \text { and } \quad A_{\theta}=A_{\theta}^{0}+\frac{\delta A_{\theta}}{\rho^{2}}+\ldots
$$

One would like to solve for $A_{\theta}^{0}, \delta \tilde{R}, \delta A_{t}$, and $\delta A_{\theta}$ in terms of the homogeneous solutions.

The equations become

$$
\begin{aligned}
& \partial_{z}\left(f \partial_{z} A_{\theta}^{0}\right)-\left(\tilde{R}^{0}\right)^{2}\left(A_{\theta}^{0}-n\right) \\
& +\frac{1}{\rho^{2}}\left(\partial_{z}\left(f \partial_{z} \delta A_{\theta}\right)-\left(\tilde{R}^{0}\right)^{2} \delta A_{\theta}-2 \tilde{R}^{0} A_{\theta}^{0} \delta \tilde{R}\right)+\ldots=0 \\
& \left(\partial_{z}\left(f \partial_{z} \delta \tilde{R}\right)-\left(z-\frac{\left(A_{t}^{0}\right)^{2}}{f}\right) \delta \tilde{R}\right) \\
& \quad+\frac{2 \tilde{R}^{0} A_{t}^{0}}{f} \delta A_{t}-\left(A_{\theta}^{0}-n\right)^{2} \tilde{R}^{0}+\ldots=0 \\
& \left(f \partial_{z}^{2} \delta A_{t}-\tilde{R}_{0}^{2} \delta A_{t}\right)-2 \tilde{R}^{0} A_{t}^{0} \delta \tilde{R}+\ldots=0 .
\end{aligned}
$$

Equating the coefficients of powers of $\rho$ gives

$$
\begin{aligned}
& \partial_{z}\left(f \partial_{z} A_{\theta}^{0}\right)-\left(\tilde{R}^{0}\right)^{2}\left(A_{\theta}^{0}-n\right)=0 \\
& \partial_{z}\left(f \partial_{z} \delta A_{\theta}\right)-\left(\tilde{R}^{0}\right)^{2} \delta A_{\theta}-2 \tilde{R}^{0} A_{\theta}^{0} \delta \tilde{R}=0 \\
& \partial_{z}\left(f \partial_{z} \delta \tilde{R}\right)-\left(z-\frac{\left(A_{t}^{0}\right)^{2}}{f}\right) \delta \tilde{R}+\frac{2 \tilde{R}^{0} A_{t}^{0}}{f} \delta A_{t}-\left(A_{\theta}^{0}-n\right)^{2} \tilde{R}^{0}=0 \\
& f \partial_{z}^{2} \delta A_{t}-\tilde{R}_{0}^{2} \delta A_{t}-2 \tilde{R}^{0} A_{t}^{0} \delta \tilde{R}=0 .
\end{aligned}
$$

In addition to these differential equations one must also impose boundary and regularity conditions. For the scalar field we should impose the boundary conditions appropriate for the type of condensate, $\partial_{z} \delta \tilde{R}(0)=0\left(<\mathcal{O}_{1}>\right.$ case $)$ or $\delta \tilde{R}(0)=0$ $\left(<\mathcal{O}_{2}>\right.$ case $)$. For $\delta A_{t}$ we require that the chemical potential not be spatially varying, $\delta A_{t}(0)=0$. Regularity at the horizon requires $\delta A_{t}(1)=0$. To study vacuum properties we should set all external sources for the superflow to zero, $A_{\theta}^{0}(0)=$ $\delta A_{\theta}(0)=0$. 
The most important thing to note is that the partial differential equations reduce to a set of coupled ODE's, which may be solved using any standard numerical differential equations solver. We have checked that the solutions to (73) determined using Mathematica's NDSolve agree with the solutions described in Section 4.1, The difference between solutions found with the two methods is at the .1\% level (the level to which the equations are solved by Gauss-Seidel method). Because the asymptotic analysis is wholly independent of the Gauss-Seidel approach, the agreement between the two provides an independent check of the results in Section 4.1 . 


\section{References}

[1] S. S. Gubser, "Breaking an Abelian gauge symmetry near a black hole horizon," Phys. Rev. D 78, 065034 (2008) [arXiv:0801.2977 [hep-th]].

[2] S. A. Hartnoll, C. P. Herzog and G. T. Horowitz, "Building a Holographic Superconductor," Phys. Rev. Lett. 101, 031601 (2008) arXiv:0803.3295 [hepth]].

[3] C. P. Herzog, P. K. Kovtun and D. T. Son, "Holographic model of superfluidity," arXiv:0809.4870 [hep-th].

[4] P. Basu, A. Mukherjee and H. H. Shieh, "Supercurrent: Vector Hair for an AdS Black Hole," Phys. Rev. D 79, 126004 (2009) [arXiv:0809.4494 [hep-th]].

[5] V. Keranen, E. Keski-Vakkuri, S. Nowling and K. P. Yogendran, "Dark Solitons in Holographic Superfluids," Phys. Rev. D 80, 121901 (2009) arXiv:0906.5217 [hep-th]].

[6] V. Keranen, E. Keski-Vakkuri, S. Nowling and K. P. Yogendran, "Inhomogeneous Structures in Holographic Superfluids: I. Dark Solitons," arXiv:0911.1866 [hep-th].

[7] T. Albash and C. V. Johnson, "Vortex and Droplet Engineering in Holographic Superconductors," arXiv:0906.1795 [hep-th].

[8] M. Montull, A. Pomarol and P. J. Silva, "The Holographic Superconductor Vortex," arXiv:0906.2396 [hep-th].

[9] K. Maeda, M. Natsuume and T. Okamura, "Vortex lattice for a holographic superconductor," arXiv:0910.4475 [hep-th].

[10] T. Albash and C. V. Johnson, "A Holographic Superconductor in an External Magnetic Field," JHEP 0809 (2008) 121 [arXiv:0804.3466 [hep-th]].

[11] S. A. Hartnoll, C. P. Herzog and G. T. Horowitz, "Holographic Superconductors," JHEP 0812 (2008) 015 [arXiv:0810.1563 [hep-th]].

[12] T. Albash and C. V. Johnson, "Phases of Holographic Superconductors in an External Magnetic Field," arXiv:0906.0519 [hep-th].

[13] H. b. Zeng, Z. y. Fan and Z. z. Ren, "Time Reversal Symmetry Breaking Holographic Superconductor in Constant External Magnetic Field," Phys. Rev. D 80 (2009) 066001 [arXiv:0906.2323 [hep-th]]. 
[14] M. H. Dehghani, A. M. Ghezelbash and R. B. Mann, "Vortex holography," Nucl. Phys. B 625 (2002) 389 arXiv:hep-th/0105134.

[15] M. H. Dehghani, A. M. Ghezelbash and R. B. Mann, "Abelian Higgs hair for AdS-Schwarzschild black hole," Phys. Rev. D 65 (2002) 044010 arXiv:hep-th/0107224.

[16] M. Antezza, F. Dalfovo, L. P. Pitaevskii, and S. Stringari, "Dark solitons in a superfluid Fermi gas," Phys. Rev. A 76, 043610 (2007) arXiv:0706.0601].

[17] R. Sensarma, M. Randeria, and T. L. Ho, "Vortices in Superfluid Fermi Gases through the BEC to BCS Crossover," Phys. Rev. Lett. 96, 090403 (2006) arXiv:cond-mat/0510761].

[18] I. Amado, M. Kaminski and K. Landsteiner, "Hydrodynamics of Holographic Superconductors," JHEP 0905, 021 (2009) [arXiv:0903.2209 [hep-th]].

[19] J. M. Kosterlitz, D. J. Thouless, "Ordering, metastability and phase transitions in two-dimensional systems," Journal of Physics C: Solid State Physics, Vol. 6 pages 1181-1203 (1973).

[20] V. L. Berezinskii, "Destruction of Long-range Order in One-dimensional and Two-dimensional Systems having a Continuous Symmetry Group I. Classical Systems," Soviet Journal of Experimental and Theoretical Physics, Vol. 32, p.493.

[21] G. T. Horowitz and M. M. Roberts, "Holographic Superconductors with Various Condensates," Phys. Rev. D 78 (2008) 126008 [arXiv:0810.1077 [hep-th]].

[22] C. P. Herzog, "Lectures on Holographic Superfluidity and Superconductivity," J. Phys. A 42 (2009) 343001 [arXiv:0904.1975 [hep-th]].

[23] S. A. Hartnoll, "Lectures on holographic methods for condensed matter physics," Class. Quant. Grav. 26, 224002 (2009) [arXiv:0903.3246 [hep-th]].

[24] S. S. Gubser, C. P. Herzog, S. S. Pufu and T. Tesileanu, "Superconductors from Superstrings," Phys. Rev. Lett. 103 (2009) 141601 [arXiv:0907.3510 [hep-th]].

[25] J. P. Gauntlett, J. Sonner and T. Wiseman, "Holographic superconductivity in M-Theory," Phys. Rev. Lett. 103, 151601 (2009) [arXiv:0907.3796 [hep-th]].

[26] S. S. Gubser, S. S. Pufu and F. D. Rocha, "Quantum critical superconductors in string theory and M-theory," Phys. Lett. B 683 (2010) 201 arXiv:0908.0011 [hep-th]]. 
[27] J. Gauntlett, J. Sonner and T. Wiseman, "Quantum Criticality and Holographic Superconductors in M-theory," arXiv:0912.0512 [hep-th].

[28] F. Denef and S. A. Hartnoll, "Landscape of superconducting membranes," Phys. Rev. D 79, 126008 (2009) arXiv:0901.1160 [hep-th]].

[29] D. T. Son, "Low-energy quantum effective action for relativistic superfluids," arXiv:hep-ph/0204199.

[30] M. A. Valle, "Hydrodynamic fluctuations in relativistic superfluid," Phys. Rev. D 77, 2, (2008) [arxiv:hep-ph/0707.2665].

[31] F. Dalfovo, S. Giorgini, L. Pitaevskii, S. Stringari "Theory of BoseEinstein condensation in trapped gases," Rev. Mod. Phys. 71, (1999) [arxi:cond-mat/9806038].

[32] G. H. Derrick, "Comments on Nonlinear Wave Equations as Models for Elementary Particles," J. Math. Phys. 5, 1252 (1964).

[33] R. B. Diener, R. Sensarma, M. Randeria, "Quantum fluctuations in the superfluid state of the BCS-BEC crossover," Phys. Rev. A 77, 023626 (2008) arXiv:0709.2653 [cond-mat.other]].

[34] C. P. Herzog and A. Yarom, "Sound modes in holographic superfluids," Phys. Rev. D 80 (2009) 106002 arXiv:0906.4810 [hep-th]].

[35] T. Nishioka, S. Ryu and T. Takayanagi, "Holographic Superconductor/Insulator Transition at Zero Temperature," arXiv:0911.0962 [hep-th].

[36] J. Pearl, Appl. Phys. Lett. 5, 65 (1964).

[37] D. R. Nelson, "Defects in Superfluids, Superconductors and Membranes," [arXiv:9502114v1 [cond-mat]].

[38] J. W. Chen, Y. J. Kao and W. Y. Wen, "Peak-Dip-Hump from Holographic Superconductivity," arXiv:0911.2821 [hep-th].

[39] T. Faulkner, G. T. Horowitz, J. McGreevy, M. M. Roberts and D. Vegh, "Photoemission 'experiments' on holographic superconductors," arXiv:0911.3402 [hep-th].

[40] S. S. Gubser, F. D. Rocha and P. Talavera, "Normalizable fermion modes in a holographic superconductor," arXiv:0911.3632 [hep-th].

[41] S. Nakamura, H. Ooguri and C. S. Park, "Gravity Dual of Spatially Modulated Phase," arXiv:0911.0679 [hep-th]. 
[42] O. C. Umeh, "Scanning the Parameter Space of Holographic Superconductors," JHEP 0908 (2009) 062 [arXiv:0907.3136 [hep-th]].

[43] Y. Kim, Y. Ko and S. J. Sin, "Density driven symmetry breaking and Butterfly effect in holographic superconductors," Phys. Rev. D 80 (2009) 126017 arXiv:0904.4567 [hep-th]].

[44] Y. Nishida and H. Abuki, "BCS-BEC crossover in relativistic superfluid and its possible realization in QCD," Phys. Rev. D 72 (2005) 096004 arXiv:hep-ph/0504083.

[45] D. T. Son, "Toward an AdS/cold atoms correspondence: a geometric realization of the Schroedinger symmetry," Phys. Rev. D 78, 046003 (2008) arXiv:0804.3972 [hep-th]].

[46] K. Balasubramanian and J. McGreevy, "Gravity duals for non-relativistic CFTs," Phys. Rev. Lett. 101, 061601 (2008) [arXiv:0804.4053 [hep-th]].

[47] S. Kachru, X. Liu and M. Mulligan, "Gravity Duals of Lifshitz-like Fixed Points," Phys. Rev. D 78, 106005 (2008) [arXiv:0808.1725 [hep-th]].

[48] E. J. Brynjolfsson, U. H. Danielsson, L. Thorlacius and T. Zingg, "Holographic Superconductors with Lifshitz Scaling," arXiv:0908.2611 [hep-th].

[49] O. Aharony, S. S. Gubser, J. M. Maldacena, H. Ooguri and Y. Oz, "Large N field theories, string theory and gravity," Phys. Rept. 323, 183 (2000) arXiv:hep-th/9905111. 